\title{
Computational experiment on determination of load on the rollers of continuous casting machine
}

\author{
Navak Bashirov $^{1}$, Daniya Zaripova ${ }^{2}$ and Irina Bashirova ${ }^{1}$ \\ ${ }^{1}$ Vologda State University, Institute of civil engineering, 160000 Vologda Lenina st. 15, Russia \\ ${ }^{2}$ Kazan State Power Engineering University, Kazan, Russia
}

\begin{abstract}
The article is devoted to calculations of load on rollers of a curvilinear continuous casting machine during bending of the ingot in the levelling section. The calculation takes into account the elastic and plastic characteristics of metal and the flexibility of the roller apparatus. Determination of load on rollers together with meeting other requirements such as reducing the cooling water consumption, minimizing the energy consumption enables optimizing the technology of slab cooling in the secondary cooling zone (SCZ) and obtaining metal of the required quality.
\end{abstract}

\section{Introduction}

Currently, advanced continuous casting machines (CCM) are used for metal casting. In curvilinear-radial CCM, the most loaded section of the technological axis of machine is the levelling section, where the slab is bent. Here an ingot is straightened from a radial shape to a straight line and it is moved to a horizontal plane. Calculation of loads due to levelling is a complex and challenging procedure both from mathematical and technological points of view as many parameters of the machine and cast metal must be taken into account. These are the plastic and elastic properties of the slab metal itself, which also change both along the slab cross section and along the technological axis of the machine; compliance (displacement) of the roller apparatus itself due to the enormous forces and moments of forces acting during bending.

The mathematical model for calculating the load on rollers at a constant speed of slab withdrawal, i.e. when the load on slab during its straightening in each roller, the rigidity of the slab metal, and the thickness of the formed slab do not change in time, was considered in [1]. In this paper the differential equations with $4 \mathrm{n}$ boundary conditions were solved using the iterative method. At the first stage, the position of the bent slab axis is found from deflection of rollers. At the second stage, the deflection of rollers is found from the position of the bent axis. Since these differential equations are not solved directly, a whole series of techniques of both mathematical and computational nature was used to solve the problem.

In [1], the load on rollers was found by a modification of mathematical model, where the curvature of the elastic component of the technological path $y^{\prime \prime}(x)$ was adopted instead of the true curvature $y_{y}^{\prime \prime}(x) /\left(1+\left(y_{y}^{\prime}(x)^{2}\right)^{0.5}\right.$.

It is impossible or difficult to determine the load on rollers from field experiments and by construction a physical model based on them, since there are several tens of pairs of rollers on the levelling section. Therefore, it is more affordable to determine the load on CCM rollers from computational experiment. Firstly, it is based on creation of a mathematical model of object under study, and secondly, when conducting it, the initial mathematical model for calculating the load on rollers must undergo a series of transformations. These transformations, ultimately, should lead to such a computational algorithm that can be implemented on a computer. The ways of transforming mathematical models are directly related to mathematical methods that are used for the quantitative analysis of these models. Methods of transforming mathematical models of different levels (meta-level, micro-level) are given in [2]. Next, computer calculations, processing and analysis of the obtained results are carried out.

The purpose of this study is to determine the load on rollers at the levelling section based on the methods of computational experiment by creating a mathematical model of object using iteration method.

\section{Materials and methods}

Consider sequentially the main stages of computational experiment for determining the load on rollers at the levelling section of CCM with constant stationary casting of metal [3].

1. Construction of mathematical model for determining the load on rollers. Figure 1 shows a model of displacements (trajectories) along the technological 


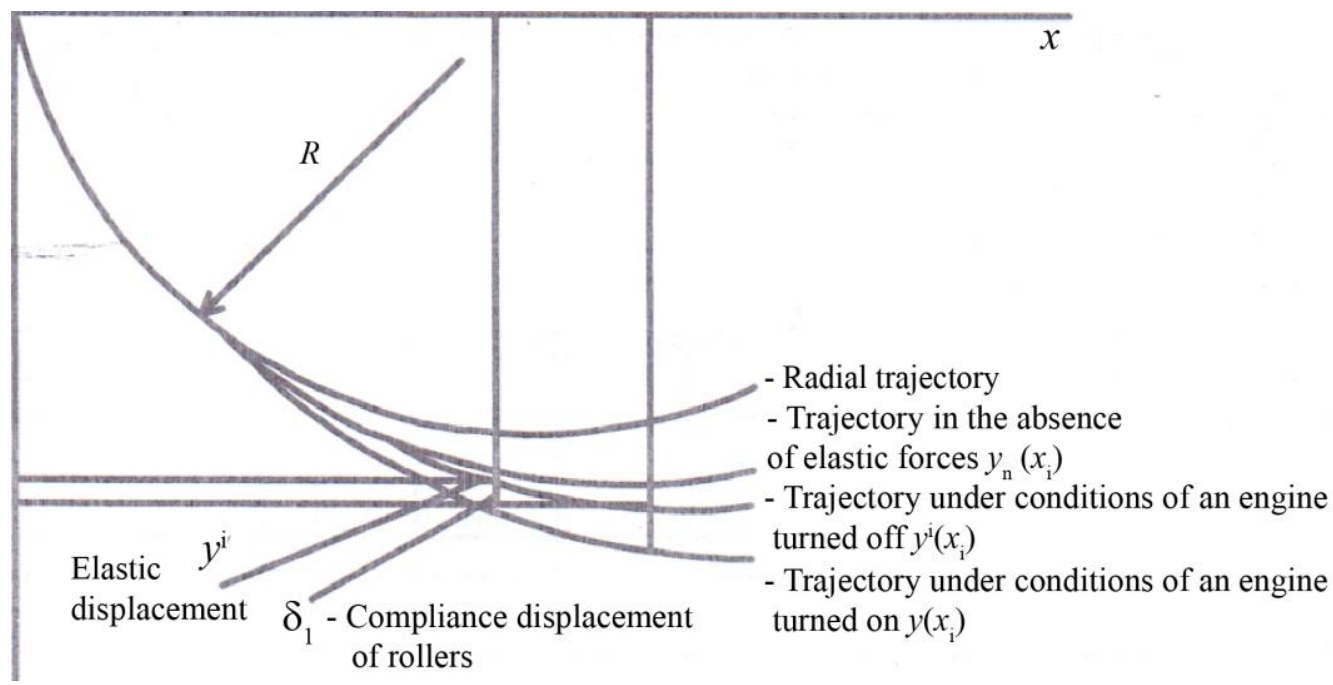

Fig. 1. Trajectories of technological axis during straightening of the ingot at CCM.

axis in slab during straightening of the ingot at CCM in the presence (absence) of various casting parameters.

The trajectories are described as follows:

$$
\left\{\begin{array}{l}
\frac{y_{y}^{\prime \prime}(x)}{\left(1+\left(y_{y}^{\prime}(x)\right)^{2}\right)^{\frac{3}{2}}}-z(x)=0, \\
z^{\prime \prime}(x)=0, \quad x_{i-1}<x<x_{i}, \\
y_{n}^{\prime \prime \prime}(x)=\frac{1}{v}\left(\frac{p(x)}{g(x)} \cdot y_{y}^{\prime \prime}(x)\right)^{5}, \quad x_{i-1}<x<x_{i}, \quad i=1,2, \ldots, n,
\end{array}\right.
$$

and meet the levelling conditions at points $x_{i}$ :

$$
\begin{aligned}
& y_{y}\left(x_{i}\right)+y_{y}\left(x_{i}\right)+k_{i}\left(\frac{z^{\prime}\left(x_{i}+0\right)}{g\left(x_{i}+0\right)}-\frac{z^{\prime}\left(x_{i}-0\right)}{g\left(x_{i}-0\right)}\right)=y_{i} \\
& i=0,1, \ldots n \\
& \frac{z\left(x_{i}+0\right)}{g\left(x_{i}+0\right)}-\frac{z\left(x_{i}-0\right)}{g\left(x_{i}-0\right)}=0, \\
& i=1,2, \ldots, n-1, \\
& z\left(x_{0}+0\right)=0, \\
& z\left(x_{n}+0\right)=0,
\end{aligned}
$$

where $\left(x_{i}, y_{i}\right)$ are the coordinates of rollers from 0 -th to $n$-th; $k_{i}$ is the roller compliance coefficient, $\mathrm{mm} / \mathrm{MN}$; $g(x), p(x)$ are the elastic and plastic functions at each interval $\left(x_{i-1}, x_{i}\right)$ of the slab section characteristic; $v$ is the slab casting rate. The following notations are also used: $n+1$ is the amount of rollers; $z(x)$ is the curvature corresponding to elastic deformation caused by external forces. The figure shows that the relations between the displacements are written by the following equations:

$$
\begin{aligned}
& y_{n}\left(x_{i}\right)+y_{y}\left(x_{i}\right)=y\left(x_{i}\right) \\
& y^{i}\left(x_{i}\right)+\delta^{i}=y\left(x_{i}\right) \\
& y_{y}\left(x_{i}\right)=y^{i}\left(x_{i}\right)+\delta^{i}-y_{n}\left(x_{i}\right) \\
& y_{y}\left(x_{i}\right)=y_{y}^{i}+\delta^{i},
\end{aligned}
$$

where

$\delta^{i}$ is the compliance displacement of the $i$-th roller;

$y_{n}\left(x_{i}\right)$ is the coordinate of the technological axis of the slab as a function of plastic displacement of the $i$-th roller;

$y_{y}\left(x_{i}\right)$ is the sum of displacements from the plastic and compliance displacements of the slab of the $i$-th roller;

$y\left(x_{i}\right)$ is the coordinate of the technological axis of the machine in working condition of the $i$-th roller;

$y_{y}^{i}$ is the displacement of the technological axis due to elastic forces;

$y^{i}\left(x_{i}\right)$ is the coordinate of the technological axis of the machine at rest of the $i$-th roller.

The conditions (2) assume that the values $z_{e}^{\prime}\left(x_{0}-0\right)$ and $z^{\prime}\left(x_{n}+0\right)$ are equal to zero: $z^{\prime}\left(x_{0}-0\right)=0, z^{\prime}\left(x_{n}-0\right)=0$.

In addition, we assume that functions $y_{n}(x)$ and $y_{y}(x)$ are continuous and have continuous derivatives $y_{n}^{\prime}(x), \quad y_{y}^{\prime \prime}(x)$ and $y_{y}^{\prime}(x)$ when crossing the rollers:

$$
\begin{aligned}
& y_{n}^{(j)}\left(x_{i}-0\right)=y_{n}^{(j)}\left(x_{i}+0\right) \\
& i=1,2, \ldots, n-1 ; j=0,1,2
\end{aligned}
$$




$$
\begin{aligned}
& y_{y}^{(j)}\left(x_{i}-0\right)=y_{y}^{(j)}\left(x_{i}+0\right) \\
& i=1,2, \ldots, n-1 ; j=0,1 .
\end{aligned}
$$

Besides this, the function $y_{n}(x)$ at $x=x_{0}$ meets the following initial conditions:

$$
y_{n}\left(x_{0}\right)=y_{n 0}, y_{n}^{\prime}\left(x_{0}\right)=y_{n 1}, y_{n}^{\prime \prime}\left(x_{0}\right)=\frac{1}{R},
$$

where $y_{n 0}=y_{0}$ is the coordinate of the zero roller, $y_{n 1}$ is the angle of inclination of the tangent to slab at the point of contact with the zero roller, $R$ is the radius of curvature of a CCM section.

2. The algorithm for calculating the load on straightening section, taking into account the full curvature formula, will be as follows.

To obtain the algorithm, the initial mathematical model must undergo a series of transformations. These transformations, ultimately, should lead to such an algorithm that can be implemented on computer. The ways of transforming mathematical models are directly related to mathematical methods that are used for the quantitative analysis of these models. Methods of transforming mathematical models of different levels (meta-level, micro-level) are given in [2].

In this paper, we consider two ways of converting a mathematical model for calculating the load on rollers at the CCM straightening section for the quasi-stationary mode of metal casting. The mathematical model of loading the CCM roller apparatus, which takes into account the elastic and plastic properties of the slab material, varying both in the cross section of the slab and along the technological axis of machine, as well as flexibility of the roller apparatus itself, which has the ability to redistribute the loads on these rollers, is given in [2].

\section{Results and discussion}

The procedure for solving differential equations with $4 \mathrm{n}$ boundary conditions in [2] was performed using the iterative method. The calculation algorithm was as follows: at the first stage, the position of the bent axis of slab $y_{y}(x)$ is found from deflection of rollers $y_{y}^{i}(x)$, $i=0,1, \ldots n$. Formally, this procedure is designated as applying the operator $F$ to vector $\left\{y_{y}^{i}(x)\right\}$ : $y_{y}(x)=F\left[\left\{y_{y}^{i}\right\}\right]$. At the second stage, the deflection of rollers $y_{y}^{i}(x)$ are found from position of the bent axis. Formally, this procedure is designated as applying the operator $H$ to function $y_{y}(x):\left\{y_{y}^{i}\right\}=H\left[\left\{y_{y}(x)\right\}\right]$.

To find the deflection of rollers from the curvilinear path $y_{y}^{i}(x)$, the first step is to find the trajectory of the points of the neutral axis by the ratio of elastic and plastic properties of slab metal, provided there are no external forces

$$
\begin{aligned}
& y_{n}(x)=y_{n}\left(x_{0}\right)+y_{n}^{\prime}\left(x_{0}\right)\left(x-x_{0}\right)+ \\
& +y_{n}^{\prime \prime}\left(x_{0}\right) \frac{\left(x-x_{0}\right)^{2}}{2}+\frac{1}{2 \cdot v} \int_{x_{0}}^{x}(x-s) \cdot\left(\frac{p(s)}{g(s)} \cdot z(s)\right)^{5} d s
\end{aligned}
$$

The second step is to determine from the condition $y_{y}^{i}=y^{i}\left(x_{i}\right)-y_{n}^{i}$ the deflection of rollers from the known coordinates of rollers $y^{i}\left(x_{i}\right)$ of the idle machine by introducing the deflection parameter of rollers in the presence of external elastic forces.

To solve the problem, the authors used a whole series of mathematical and computational techniques and $4 n$ boundary conditions.

In [1], the load on rollers was found by a modification of mathematical model, where the curvature of the elastic component of the technological path $y^{\prime \prime}(x)$ was adopted instead of the true curvature $y_{y}^{\prime \prime}(x) /\left(1+\left(y_{y}^{\prime}(x)^{2}\right)^{0.5}\right.$. To calculate the load on rollers and find the bending moment in this work, only $2 n+2$ boundary conditions are needed. One of the options for algorithmization of mathematical model for calculating the load on rollers in the straightening section can be as follows.

1. The matrix of parameters of all rollers and characteristics of metal are formed on the straightening section: $\left(x_{i}, y_{i}\right)$ are the coordinates of rollers from 0 -th to $n$-th; $k_{i}$ is the roller compliance coefficient, $\mathrm{mm} / \mathrm{MN}$; $g(x), p(x)$ are the elastic and plastic properties of metal, continuously limited functions on each interval $\left(x_{i-1}, x_{i}\right) ; v$ is the slab rate; $n+1$ is the amount of rollers.

2. The coefficients of linear function $z(x)=A_{i} \cdot\left(x-x_{i}\right)+B_{i}$ of the general solution of the differential equation $z^{\prime \prime}\left(x_{i}\right)=0, \quad x_{i-1}<x<x_{i}$, $i=1,2, \ldots, n$, are set at each interval of straightening: $A, A_{1}, A_{2}, \ldots, A_{n}, A_{n+1}, B_{1}, B_{2}, \ldots, B_{n}$.

3. The equation

$y_{y}(x)=A_{0}+A_{n+1} \cdot\left(x-x_{0}\right)+\int_{x_{0}}^{x}(x-s) \cdot z(s) d s, \quad x_{0}<x<x_{n}$, and the set coefficients are used to determine the elastic deflection of rollers $y_{y}(x)$ at each interval of straightening.

4. The relation $\eta_{i}=y_{y}\left(x_{i}\right)-y_{y}\left(x_{i}\right)-k_{i}\left(\frac{y_{e}^{\prime \prime \prime}\left(x_{i}+0\right)}{g\left(x_{i}+0\right)}-\frac{y_{e}^{\prime \prime \prime}\left(x_{i}-0\right)}{g\left(x_{i}-0\right)}\right)$, $i=0,1, \ldots n$ is calculated using the preset coefficients at each interval.

5. The system of equations is solved

$$
\left\{\begin{array}{l}
y_{n}\left(x_{i}\right)=\eta_{i}, \quad x_{i-1}<x<x_{i}, \\
B=C \cdot A, \quad x_{i-1}<x<x_{i}, \quad i=1,2, \ldots, n,
\end{array}\right.
$$


and the coefficients for each interval of the technological axis are determined $A, B, A_{1}, A_{2}, \ldots, A_{n}$.

$$
\begin{array}{cccc}
6 . \text { Item } & \text { is } & \text { repeated } & \text { and } \\
y_{y}(x)=A_{0}+A_{n+1} \cdot\left(x-x_{0}\right)+\int_{x_{0}}^{x}(x-s) \cdot z(s) d s, & x_{0}<x<x_{n}
\end{array}
$$

are determined using newly calculated coefficients

$A, \quad B, \quad A_{1}, \quad A_{2}, \ldots, A_{n}$.

7. The

relation

$\eta_{i}=y_{y}\left(x_{i}\right)-y_{y}\left(x_{i}\right)-k_{i}\left(\frac{y_{y}^{\prime \prime \prime}\left(x_{i}+0\right)}{g\left(x_{i}+0\right)}-\frac{y_{y}^{\prime \prime \prime}\left(x_{i}-0\right)}{g\left(x_{i}-0\right)}\right)$,

$i=0,1, \ldots n$ is calculated using the newly obtained coefficients.

8. Thus, the iteration continues until sufficient computational accuracy is achieved.

By substituting the following data: slab width $b=1450 \mathrm{~mm}$, slab thickness $a=250 \mathrm{~mm}$, surface temperature of upper edge $T_{u s}=1100^{\circ} \mathrm{C}$, surface temperature of lateral edge $T_{l s}=1000^{\circ} \mathrm{C}$ and performing the calculation, we get $g_{42}=1.071 \cdot 10^{-14} \frac{1}{\mathrm{~N} \cdot \mathrm{mm}^{2}}, \quad g_{43}=1.062 \cdot 10^{-14} \frac{1}{\mathrm{~N} \cdot \mathrm{mm}^{2}}$, $g_{44}=1.054 \cdot 10^{-14} \frac{1}{\mathrm{~N} \cdot \mathrm{mm}^{2}}, \quad g_{45}=1.046 \cdot 10^{-14} \frac{1}{\mathrm{~N} \cdot \mathrm{mm}^{2}}$, $g_{46}=1.038 \cdot 10^{-14} \frac{1}{\mathrm{~N} \cdot \mathrm{mm}^{2}}, \quad g_{47}=1.021 \cdot 10^{-14} \frac{1}{\mathrm{~N} \cdot \mathrm{mm}^{2}}$. And we obtain: $p_{42}=3.894 \cdot 10^{-10}\left(\mathrm{~N} \cdot \mathrm{mm}^{0.5} \cdot \mathrm{s}^{0.2}\right)^{-1}$, $p_{42}=3.855 \cdot 10^{-10}\left(\mathrm{~N} \cdot \mathrm{mm}^{0.5} \cdot \mathrm{s}^{0.2}\right)^{-1}$, $p_{42}=3.818 \cdot 10^{-10}\left(\mathrm{~N} \cdot \mathrm{mm}^{0.5} \cdot \mathrm{s}^{0.2}\right)^{-1}$, $p_{42}=3.784 \cdot 10^{-10}\left(\mathrm{~N} \cdot \mathrm{mm}^{0.5} \cdot \mathrm{s}^{0.2}\right)^{-1}$, $p_{42}=3.751 \cdot 10^{-10}\left(\mathrm{~N} \cdot \mathrm{mm}^{0.5} \cdot \mathrm{s}^{0.2}\right)^{-1}$, $p_{42}=3.722 \cdot 10^{-10}\left(\mathrm{~N} \cdot \mathrm{mm}^{0.5} \cdot \mathrm{s}^{0.2}\right)^{-1}$.

The roller compliance coefficients $k \mathrm{i}$ taking into account the slab width $b=1450 \mathrm{~mm}$, the roller elastic modulus $E=0.5 \frac{\mathrm{MN}}{\mathrm{mm}^{2}}$, its length $l_{p}=2500 \mathrm{~mm}$, outer $D_{p}=380 \mathrm{~mm}$, and inner diameters $d_{p}=100$, $k_{42}=k_{43}=k_{44}=k_{45}=k_{46}=k_{47}=0.225 \mathrm{~mm} / \mathrm{MN}$

Knowing these parameters, the force on rollers and bending moments are determined. Further some calculated values are presented. For calculation, the levelling section of CCM with a base radius of $\mathrm{R}=$ $10000 \mathrm{~mm}$ was considered. Metal was casted into slabs with a thickness of $250 \mathrm{~mm}$, a width of $1700 \mathrm{~mm}$, and a casting speed of $1.2 \mathrm{~m} / \mathrm{min}$ with a flexibility of a roller apparatus of $0.3 \mathrm{~mm} / \mathrm{MN}$ and levelling step of $312 \mathrm{~mm}$. The calculated loads on rollers are the following: roller
No.34 $F=0.21 \mathrm{MN}$, roller No.39 $F=0.76 \mathrm{MN}$; roller No. $43 F=1.33 \mathrm{MN}$.

The initial results are obtained after the $k$-th iteration, depending on the accuracy of the task. The initial approximations should be chosen as close as possible to the true solution, since if the initial approximations are poorly defined, it is impossible to solve the system of equations.

\section{Conclusions}

As a result of the studies, an algorithm was developed for determining the load on the rollers in the secondary cooling zone of CCM during ingot straightening based on a computational experiment using the iteration method. Using the created mathematical model of levelling and a computational experiment, the loads on the rollers are determined when the slab is straightened in a continuous casting machine.

\section{References}

[1] N.G. Bashirov, E.M. Mukhamadiev, A.N. Naimov, A mathematical model for calculating the load on rollers at the levelling section of a curvilinear CCM, University science - to the region: Materials of the Tenth All-Russian Scientific and Technical Conference, in 2 vols, vol. 1 (Vologda: VSTU, 3-5, 2012).

[2] L.V. Bulanov, L.G. Korzunin, E.P. Parfyonov, N.A. Yurovsky, Continuous casting machines (Kazan, 349, 2003).

[3] V.S. Zarubin, Mathematical modelling in technology: textbook for universities, 3-ed. (Moscow: Publishing House of MSTU, 495, 2010). 